PROCEEDINGS OF THE

AMERICAN MATHEMATICAL SOCIETY

Volume 136, Number 12, December 2008, Pages 4135-4137

S 0002-9939(08)09427-6

Article electronically published on June 11, 2008

\title{
MINIMAL GENERATORS FOR SYMMETRIC IDEALS
}

\author{
CHRISTOPHER J. HILLAR AND TROELS WINDFELDT
}

(Communicated by Bernd Ulrich)

\begin{abstract}
Let $R=K[X]$ be the polynomial ring in infinitely many indeterminates $X$ over a field $K$, and let $\mathfrak{S}_{X}$ be the symmetric group of $X$. The group $\mathfrak{S}_{X}$ acts naturally on $R$, and this in turn gives $R$ the structure of a module over the group ring $R\left[\mathfrak{S}_{X}\right]$. A recent theorem of Aschenbrenner and Hillar states that the module $R$ is Noetherian. We address whether submodules of $R$ can have any number of minimal generators, answering this question positively.
\end{abstract}

Let $R=K[X]$ be the polynomial ring in infinitely many indeterminates $X$ over a field $K$. Write $\mathfrak{S}_{X}\left(\right.$ resp. $\left.\mathfrak{S}_{N}\right)$ for the symmetric group of $X$ (resp. $\left.\{1, \ldots, N\}\right)$ and $R\left[\mathfrak{S}_{X}\right]$ for its (left) group ring, which acts naturally on $R$. A symmetric ideal $I \subseteq R$ is an $R\left[\mathfrak{S}_{X}\right]$-submodule of $R$.

Aschenbrenner and Hillar recently proved [1] that all symmetric ideals are finitely generated over $R\left[\mathfrak{S}_{X}\right]$. They were motivated by finiteness questions in chemistry [3] and algebraic statistics [2]. In proving the Noetherianity of $R$, it was shown that a symmetric ideal $I$ has a special, finite set of generators called a minimal Gröbner basis. However, the more basic question of whether $I$ is always cyclic (already asked by Josef Schicho 4]) was left unanswered in 1]. Our result addresses a generalization of this important issue.

Theorem 1. For every positive integer $n$, there are symmetric ideals of $R$ generated by $n$ polynomials which cannot have fewer than $n R\left[\mathfrak{S}_{X}\right]$-generators.

In what follows, we work with the set $X=\left\{x_{1}, x_{2}, x_{3}, \ldots\right\}$, although as remarked in [1, this is not really a restriction. In this case, $\mathfrak{S}_{X}$ is naturally identified with $\mathfrak{S}_{\infty}$, the permutations of the positive integers, and $\sigma x_{i}=x_{\sigma i}$ for $\sigma \in \mathfrak{S}_{\infty}$.

Let $M$ be a finite multiset of positive integers and let $i_{1}, \ldots, i_{k}$ be the list of its distinct elements, arranged so that $m\left(i_{1}\right) \geq \cdots \geq m\left(i_{k}\right)$, where $m\left(i_{j}\right)$ is the multiplicity of $i_{j}$ in $M$. The type of $M$ is the vector $\lambda(M)=\left(m\left(i_{1}\right), m\left(i_{2}\right), \ldots, m\left(i_{k}\right)\right)$. For instance, the multiset $M=\{1,1,1,2,3,3\}$ has type $\lambda(M)=(3,2,1)$. Multisets are in bijection with monomials of $R$. Given $M$, we can construct the monomial:

$$
\mathbf{x}_{M}^{\lambda(M)}=\prod_{j=1}^{k} x_{i_{j}}^{m\left(i_{j}\right)} .
$$

Conversely, given a monomial, the associated multiset is the set of indices appearing in it, along with multiplicities. The action of $\mathfrak{S}_{\infty}$ on monomials coincides with the

Received by the editors September 6, 2006, and, in revised form, October 25, 2007.

2000 Mathematics Subject Classification. Primary 13E05, 13E15, 20B30, 06A07.

Key words and phrases. Invariant ideal, symmetric group, Gröbner basis, minimal generators.

The work of the first author was supported under an NSF Postdoctoral Fellowship. 
natural action of $\mathfrak{S}_{\infty}$ on multisets $M$, and this action preserves the type of a multiset (resp. monomial). We also note the following elementary fact.

Lemma 2. Let $\sigma \in \mathfrak{S}_{\infty}$ and $f \in R$. Then there exists a positive integer $N$ and $\tau \in \mathfrak{S}_{N}$ such that $\tau f=\sigma f$.

Theorem 1 is a direct corollary of the following result.

Theorem 3. Let $G=\left\{g_{1}, \ldots, g_{n}\right\}$ be a set of monomials of degree $d$ with distinct types and fix a matrix $C=\left(c_{i j}\right) \in K^{n \times n}$ of rank $r$. Then the submodule $I=\left\langle f_{1}, \ldots, f_{n}\right\rangle_{R\left[\mathfrak{S}_{\infty}\right]} \subseteq R$ generated by the $n$ polynomials, $f_{j}=\sum_{i=1}^{n} c_{i j} g_{i}$ $(j=1, \ldots, n)$, cannot have fewer than $r R\left[\mathfrak{S}_{\infty}\right]$-generators.

Proof. Suppose that $p_{1}, \ldots, p_{k}$ are generators for $I$; we prove that $k \geq r$. Since each $p_{l} \in I$, it follows that each is a linear combination, over $R\left[\mathfrak{S}_{\infty}\right]$, of monomials in $G$. Therefore, each monomial occurring in $p_{l}$ has degree at least $d$, and, moreover, any degree $d$ monomial in $p_{l}$ has the same type as one of the monomials in $G$.

Write each of the monomials in $G$ in the form $g_{i}=\mathbf{x}_{M_{i}}^{\lambda_{i}}$ for multisets $M_{1}, \ldots, M_{n}$ with corresponding distinct types $\lambda_{1}, \ldots, \lambda_{n}$, and express each generator $p_{l}$ as

$$
p_{l}=\sum_{i=1}^{n} \sum_{\lambda(M)=\lambda_{i}} u_{i l M} \mathbf{x}_{M}^{\lambda_{i}}+q_{l}
$$

in which $u_{i l M} \in K$ with only finitely many of them nonzero, each monomial in $q_{l}$ has degree larger than $d$, and the inner sum is over multisets $M$ with type $\lambda_{i}$.

Since each polynomial in $\left\{f_{1}, \ldots, f_{n}\right\}$ is a finite linear combination of the $p_{l}$, and since only finitely many integers are indices of monomials appearing in $p_{1}, \ldots, p_{k}$, we may pick $N$ large enough so that all of these linear combinations can be expressed with coefficients in the subring $R\left[\mathfrak{S}_{N}\right]$ (cf. Lemma 2). Therefore, we have

$$
f_{j}=\sum_{l=1}^{k} \sum_{\sigma \in \mathfrak{S}_{N}} s_{l j \sigma} \sigma p_{l}
$$

for some polynomials $s_{l j \sigma} \in R$. Substituting (11) into (2) gives us that

$$
f_{j}=\sum_{l=1}^{k} \sum_{\sigma \in \mathfrak{S}_{N}} \sum_{i=1}^{n} \sum_{\lambda(M)=\lambda_{i}} v_{l j \sigma} u_{i l M} \mathbf{x}_{\sigma M}^{\lambda_{i}}+h_{j},
$$

in which each monomial appearing in $h_{j} \in R$ has degree greater than $d$ and $v_{l j \sigma}$ is the constant term of $s_{l j \sigma}$. Since each $f_{j}$ has degree $d$, we have that $h_{j}=0$. Thus,

$$
\sum_{i=1}^{n} c_{i j} \mathbf{x}_{M_{i}}^{\lambda_{i}}=\sum_{l=1}^{k} \sum_{\sigma \in \mathfrak{S}_{N}} \sum_{i=1}^{n} \sum_{\lambda(M)=\lambda_{i}} v_{l j \sigma} u_{i l M} \mathbf{x}_{\sigma M}^{\lambda_{i}} .
$$

Next, for a fixed $i$, take the sum on each side in this last equation of the coefficients of monomials with the type $\lambda_{i}$. This produces the $n^{2}$ equations

$$
c_{i j}=\sum_{l=1}^{k} \sum_{\sigma \in \mathfrak{S}_{N}} \sum_{\lambda(M)=\lambda_{i}} v_{l j \sigma} u_{i l M}=\sum_{l=1}^{k}\left(\sum_{\lambda(M)=\lambda_{i}} u_{i l M}\right)\left(\sum_{\sigma \in \mathfrak{S}_{N}} v_{l j \sigma}\right)=\sum_{l=1}^{k} U_{i l} V_{l j},
$$

in which $U_{i l}=\sum_{\lambda(M)=\lambda_{i}} u_{i l M}$ and $V_{l j}=\sum_{\sigma \in \mathfrak{S}_{N}} v_{l j \sigma}$. Set $U$ to be the $n \times k$ matrix $\left(U_{i l}\right)$ and similarly let $V$ denote the $k \times n$ matrix $\left(V_{l j}\right)$. These $n^{2}$ equations are 
represented by the equation $C=U V$, leading to the following chain of inequalities:

$$
r=\operatorname{rank}(C)=\operatorname{rank}(U V) \leq \min \{\operatorname{rank}(U), \operatorname{rank}(V)\} \leq \min \{n, k\} \leq k .
$$

Therefore, we have $k \geq r$, and this completes the proof.

\section{REFERENCES}

[1] M. Aschenbrenner and C. Hillar, Finite generation of symmetric ideals, Trans. Amer. Math. Soc. 359 (2007), 5171-5192. MR.2327026

[2] M. Drton, B. Sturmfels and S. Sullivant, Algebraic factor analysis: tetrads, pentads and beyond, Probability Theory and Related Fields 138 (2007) 463-493. MR2299716

[3] E. Ruch, A. Schönhofer and I. Ugi, Die Vandermondesche Determinante als Näherungsansatz für eine Chiralitätsbeobachtung, ihre Verwendung in der Stereochemie und zur Berechnung der optischen Aktivität, Theor. Chim. Acta 7 (1967), 420-432.

[4] J. Schicho, private communication, 2006.

Department of Mathematics, Texas A\&M University, College Station, Texas 77843

E-mail address: chillar@math.tamu.edu

Department of Mathematical Sciences, University of Copenhagen, DK-1165 Copenhagen, Denmark

E-mail address: windfeldt@math.ku.dk 